\title{
Evidencia Científica actual sobre la utilidad del Ultrasonido de Alta Intensidad (HIFU) en el tratamiento del Adenocarcinoma Prostático
}

\author{
E. Lledó García, J. Jara Rascón, D. Subirá Ríos, F. Herranz Amo, J.I. Martínez-Salamanca, \\ C. Hernández Fernández
}

Servicio de Urología. Hospital General Universitario Gregorio Marañón. Madrid.

Actas Urol Esp 2005; 29 (2): 131-137

\section{RESUMEN}

EVIDENCIA CIENTÍFICA ACTUAL SOBRE LA UTILIDAD DEL ULTRASONIDO DE ALTA INTENSIDAD (HIFU) EN EL TRATAMIENTO DEL ADENOCARCINOMA PROSTÁTICO

Objetivo: Evaluar en la literatura específica existente la evidencia científica sobre la utilización del Ultrasonido de Alta Intensidad (HIFU) en el tratamiento del adenocarcinoma prostático (Cap).

Método: Se revisa la literatura a través de tres bases de datos: PubMed, Cochrane Library, HTA database. Se seleccionaron varios artículos teniendo en cuenta el número de casos, los criterios de inclusión de los pacientes, el tiempo de seguimiento. Intentamos evaluar la mejor evidencia disponible llevando a cabo una revisión sistemática de la eficacia clínica y del coste-efectividad del HIFU en el tratamiento del Cap. Analizamos supervivencia global, supervivencia libre de enfermedad y calidad de vida, incluyendo complicaciones, efectos adversos y aceptación de la técnica.

Resultados: Los trabajos disponibles se centran en dos indicaciones principales: la aplicación del HIFU como primer escalón terapéutico y como terapia de rescate en recidivas post-radioterapia. Encontramos una gran dificultad para extraer conclusiones sobre los beneficios relativos del HIFU: ausencia de media o alta evidencia y falta de comparaciones de esta terapia emergente con los tratamientos estándar. En cuanto a resultados sobre coste-efectividad tampoco objetivamos datos que nos permitieran extraer evidencia científica de calidad media/alta sobre la técnica. La mayoría de trabajos ofrecían disparidad en la definición de supervivencia libre de enfermedad (SLE), lo que dificulta la interpretación de resultados y la extracción de conclusiones. Los criterios de inclusión fueron también heterogéneos entre los diversos autores.

Conclusiones: Actualmente la evidencia científica sobre la utilidad del HIFU en el tratamiento del adenocarcinoma prostático es de calidad baja. Entre los aspectos a destacar tenemos en cuenta su capacidad de destrucción tumoral local tanto en los casos sin terapia previa como en las recidivas post-radioterapia. No se pueden extraer, sin embargo, conclusiones a medio y largo plazo por la falta de ensayos clínicos randomizados y controlados con seguimiento suficiente para medir beneficios en términos de supervivencia global y calidad de vida (balance efectos adversos/beneficios), la ausencia de comparaciones con las terapias estándar así como la heterogeneidad de criterios de definición de la SLE.

Palabras clave: Cáncer de próstata. Ultrasonido de alta intensidad. Tratamientos mínimamente invasivos.

\section{ABSTRACT}

SCIENTIFIC EVIDENCE ON THE USE OF HIGH-INTENSITY FOCAL ULTRASOUND (HIFU) IN THE TREATMENT OF PROSTATIC CARCINOMA

Objectives: To evaluate in the literature scientific evidence on the use of High-Intensity Focal Ultrasound (HIFU) in the treatment of prostatic carcinoma (PC).

Method: Three database are searched: PubMed, Cochrane Library, HTA database. Several articles were selected taking into account number of cases, inclusion criteria, duration of follow-up period. We have evaluated the best evidence available thorugh a systematic review of clinical efficacy and cost-effectiveness of HIFU in the treatment of PC. We analized global survival, diseasefree survival, and quality of life, including complications, adversal effects and acceptance of the technique.

Results: Publications available are focused on two main indications of the therapy: first step of management of PC and salvage therapy for locally recurrent PC after external beam radiotherapy. It was very difficult to draw conclusions on the relative benefits of the HIFU: lack of high or medium quality evidence and no comparisons between this technique an standard treatments. In relation to results on cost-effectiveness, no relevant studies were identified in order to get conclusions on the quality of the treatment. Most of reports offered disparity in the definition of free survival disease concept. This fact produce some misunderstanting of results and conclusions cannot be drawn correctly. Inclusion criteria were also heterogeneous between authors.

Conclusions: No high-quality clinical evidence can be established currently on the utility of HIFU as treatment of prostatic cancer. An important fact to stress is the capacity of therapy to produce tumour necrosis both as first-step treatment and as salvage therapy. No conclusions can be drawn in the long-term due to the paucity of controlled and randomized trials with adequate follow-up to establish benefits in terms of global survival and quality of life (balance adversal effects/benefits), lack of comparisons with standard options as long as different definitions of free-survival disease.

Keywords: Prostate cancer. HIFU. Minimally invasive treatments. 


\section{INTRODUCCIÓN Y ANTECEDENTES}

Debido al importante desarrollo de las técnicas de diagnóstico precoz del cáncer de próstata en los últimos años, esta neoplasia es la más frecuentemente diagnosticada en los varones de USA $^{1}$ y el segundo tumor más frecuente en Gran Bretaña y Gales ${ }^{2}$. En un estudio reciente sobre la incidencia del Cap en la Comunidad de Madrid, la incidencia estimada de esta neoplasia ha sido calculada en 100,4 casos/100.000 hombres $^{3}$. La disponibilidad del antígeno prostático específico (PSA), que permite una sospecha de la presencia del Cap en fases asintomáticas, ha permitido en los últimos 15 años un incremento dramático en la detección de esta enfermedad.

El tratamiento del Cap localizado incluye la actitud de vigilancia expectante, la prostatectomía radical y la radioterapia ${ }^{2}$. Estas opciones pueden producir efectos secundarios no deseados, como la incontinencia urinaria y la disfunción eréctil. Actualmente se encuentran en desarrollo una serie de opciones terapéuticas que intentan combinar la mínima invasividad -con todas las ventajas que este concepto supone para el paciente- junto con la efectividad oncológica y la reducción de los efectos secundarios. Técnicas que perfeccionan la radioterapia (braquiterapia, radioterapia conformacional 3D), innovaciones tecnológicas en crioterapia, ultrasonidos de alta intensidad (HIFU). Otras alternativas, como la terapia génica, se encuentran en fases completamente precoces de su desarrollo.

El ultrasonido de alta intensidad (HIFU) es capaz de inducir necrosis coagulativa en cualquier medio no viscoelástico, como cualquier tejido biológico ${ }^{1}$. Las ondas focalizadas de ultrasonido son emitidas desde un transductor y absorbidas en un área objetivo, produciendo efectos mecánicos, cavitación y efectos térmicos sin lesionar los tejidos que se encuentran interpuestos entre el emisor y el objetivo, en el camino del ultrasonido 4 . El potencial efecto antitumoral del HIFU fue ya mostrado en el terreno experimental por Chapelon ${ }^{5}$, posteriormente también en el terreno clínico ${ }^{6}$.

\section{OBJ ETIVOS}

Revisamos en el presente artículo la mejor evidencia presente en la literatura basada en la efi- cacia clínica y el coste-efectividad del Ultrasonido de Alta Intensidad en el tratamiento del adenocarcinoma de próstata. Este análisis intenta evaluar la efectividad clínica en términos de supervivencia, supervivencia libre de enfermedad, calidad de vida, incluyendo complicaciones y efectos adversos, así como aceptación de la técnica.

\section{MATERIAL Y MÉTODO}

Procedimos a buscar en la literatura aquellas referencias correspondientes a la utilización del ultrasonido de alta intensidad en el adenocarcinoma de próstata. Utilizamos las siguientes bases de datos: PubMed, Cochrane Library, Health Technology Assessment Database Seleccionamos para su análisis una serie de trabajos basándonos en el número de casos, los criterios de inclusión de los pacientes, así como en el tiempo de seguimiento. Tuvimos también en cuenta el concepto definido de supervivencia libre de enfermedad. El mayor número de estudios correspondieron a series descriptivas de casos. Ningún ensayo clínico randomizado pudo ser evaluado.

\section{RESULTADOS Y DISCUSIÓN}

Podemos plantear globalmente que la evidencia actual sobre la utilización del ultrasonido de alta intensidad en el adenocarcinoma prostático es aún de calidad baja. El análisis de resultados sobre la eficacia clínica debe valorar, especialmente, la existencia de ensayos clínicos randomizados frente a series descriptivas de casos. Estas últimas muestran de forma característica, en general, sesgos referidos a los criterios de selección de los pacientes así como a la evaluación de los objetivos, que suelen contar con seguimientos cortos. En este aspecto tuvimos gran dificultad para extraer conclusiones sobre los beneficios relativos del HIFU: ausencia de evidencia y falta de comparaciones con los tratamientos estándar. En cuanto a la valoración de los resultados sobre coste-efectividad, para las que tomamos en cuenta estimaciones basadas en el impacto de los efectos adversos en la calidad de vida de los pacientes (QUALYS: quality adjusted life years), tampoco obtuvimos, ante la ausencia de datos, información relevante. Según Hummel ${ }^{2}$ 
de un listado de 15 posibles terapias emergentes, el HIFU se encontraría en el mismo grupo de evidencias de baja calidad que, al mismo nivel, RITA, terapia génica o la fotocoagulación (Fig. 1).

Un problema adicional con el que nos encontramos al revisar la literatura es la ausencia de criterios homogéneos para definir el concepto de supervivencia libre de enfermedad (SLE). Este aspecto es fundamental para valorar los resultados obtenidos por los distintos grupos de trabajo. Según $\mathrm{Critz}^{7}$ el análisis multivariante de factores relacionados con ausencia de enfermedad revelan que la definición de estatus libre de enfermedad utilizada para calcular la SLE es tan significativa como el PSA pre-tratamiento o la puntuación de Gleason (Tabla 1). Pueden llegar a encontrarse hasta 7 puntos de corte diferentes de PSA publicados en los últimos 3 años por autores de primera línea para definir este concepto $^{1,7-9}$. Este hecho supone un grave problema en dos aspectos: en primer lugar, la interpretación del resultado de los distintos trabajos en sus seguimientos. Por otro lado, el significado clínico real de la elevación del PSA tras un tratamiento radical por Cap. Hasta un $26 \%$ de prostatectomías radicales pueden dejar restos de tejido glandular benigno en los márgenes quirúrgicos, lo que explicaría, al menos, una gran parte de los casos de progresión de PSA hasta valores $\leq 0,3$ $\mathrm{ngrs} / \mathrm{ml}$ sin cambios en períodos de 3 años desde su detección ${ }^{10}$.

El primer bloque de trabajos evaluado se refiere a la indicación de HIFU como primera terapia. Nos encontramos con 2 artículos recientes. El primero de ellos es el Estudio Multicéntrico Europeo pu-

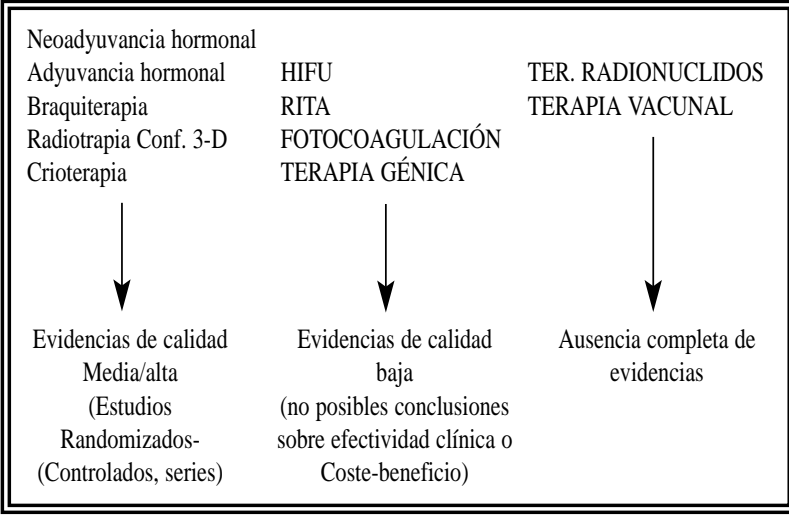

FIGURA 1. Evidencia científica de utilización de diversas opciones terapéuticas en cáncer prostático.

\section{Tabla 1}

Importancia de la definición del estatus libre de enfermedad en el porcentaje de supervivencia libre de enfermedad

Análisis multivariante de factores relacionados con ausencia de enfermedad revelan que la definición de estatus libre de enfermedad utilizada para calcular la SLE es tan significativa como el PSA pre-tratamiento o el Gleason medio

\begin{tabular}{|c|c|}
\hline Factors & pValue \\
\hline Disease-free definition & $<0.0001$ \\
\hline Pretreatment PSA & $<0.0001$ \\
\hline Gleason score* & 0.0155 \\
\hline Stage & 0.0228 \\
\hline
\end{tabular}

A STANDARD DEFINITION OF DISEASE FREEDOM IS NEEDED FOR PROSTATE CANCER: UNDETECTABLE PROSTATE SPECIFIC ANTIGEN COMPARED WITH THE AMERICAN SOCIETY OF THERAPEUTIC RADIOLOGY AND ONCOLOGY CONSENSUS DEFINITION

FRANK A. CRITZ - Radiotherapy Clinics of Georgia, Decatur. Georgia.

THE JOURNAL OF UROLOGY March 2002: 167: 1310-1313

blicado en Urology en $2004^{8}$ y cuya autoría está comandada por S. Thüroff. Este Ensayo Clínico es un fase II-III prospectivo, que evalúa la seguridad y eficacia de HIFU en el tratamiento del Cap localizado (T1-2, NO-X, MO) en pacientes no candidatos a prostatectomía radical por problemas médicos o por no deseo de someterse a los riesgos de la intervención quirúrgica. Los pacientes $(\mathrm{N}=402)$ fueron incluidos entre 1995 y 1999 en seis grandes centros europeos. La edad media fue de $69,3 \pm 7,1$ años. El volumen prostático medio era de $28 \pm 13,8$ cc y la concentración media de PSA pre-tratamiento de $10,9 \pm 8,7 \mathrm{ng} / \mathrm{ml}$ (<10:50,9\%; 10-20:3\%; >20:16\%). El 92\% de los pacientes tuvieron entre 1-4 cilindros positivos para Cap en la biopsia diagnóstica. La puntuación de Gleason fue de 2-4 en 13,2\%, 5-7 en $77,5 \%$ y $8-10$ en $9.3 \%$. Durante el seguimiento, se procedió a completar biopsias sextantes randomizadas y determinación de PSA. Cualquier biopsia positiva para Cap tras la última sesión de HIFU clasificó al paciente como fracaso terapéutico. Los pacientes recibieron una media de 1.4 sesiones de HIFU. El seguimiento medio fue de 407 días (cuartiles 1135-3598, media 321 días). $\mathrm{El}$ porcentaje de biopsias negativas observado en la población tratada fue de $87,2 \%$. El PSA tras 
tratamientos completados fue de $0,1 \mathrm{ng} / \mathrm{ml}$. Estos resultados fueron estratificados según el riesgo de la enfermedad, resultando en porcentajes de biopsia negativa de $82-92 \%$ (Tabla 2). El estudio concluye que HIFU debe ser considerada como una opción de tratamiento primario del Cap localizado, con un alto poder destructivo tumoral local. No obstante, aunque la cohorte del trabajo sea considerable, el seguimiento es extrañamente corto, el ensayo -evidentemente- no es randomizado y no queda en absoluto clara la interpretación de los valores de PSA en el seguimiento a medio plazo de los pacientes. No se refieren claramente la incidencia de complicaciones por la técnica. Es, pues, un buen trabajo aunque no permite extraer evidencias científicas de alta calidad.

\section{Tabla 2}

Porcentaje de biopsias negativas tras HIFU según riesgo relacionado del adenocarcinoma prostático

\section{ESTUDIO EUROPEO MULTICÉNTRICO}

Riesgo relacionado CaP

Porcentaje biopsias negativas

Pacientes bajo riesgo

$92,1 \%$

T1-T2a y PSA $\leq 10 \mathrm{ng} / \mathrm{ml}$ y Gleason store $\leq 6$

Pacientes riesgo intermedio

T2b o PSA $10,1-20 \mathrm{ng} / \mathrm{ml}$ o Gleason store $=7$

$86,4 \%$

Pacientes alto riesgo

$82,1 \%$

T2c o PSA $>20 \mathrm{ng} / \mathrm{ml}$ o Gleason store $\geq 8$

El segundo trabajo seleccionado es una serie descriptiva de casos, provenientes de un solo centro. Ha sido publicado recientemente en Urology por Andreas Blana, del Departamento de Urología de Regensburg, en Alemania ${ }^{1}$. En este trabajo se analizan los efectos de HIFU en una serie de 146 pacientes diagnosticados mediante biopsia transrectal de Cap T1-T2 NO MO. Todos los pacientes tenían un PSA de $\leq 15 \mathrm{ng} / \mathrm{ml}$ y una puntuación de Gleason $\leq 7$, que fueron establecidos como parte de los criterios de inclusión. Los individuos no fueron candidatos a prostatectomía radical, bien por comorbilidad o por falta de deseo de ser sometidos a la intervención. El seguimiento medio fue de 22,5 meses (4-62) e incluyó determinaciones de PSA y biopsias sex- tantes de control. En cuanto a los resultados obtenidos, el PSA nadir determinado 3 meses tras el tratamiento fue de $0,07 \mathrm{ng} / \mathrm{ml} \quad(0-5,67$ $\mathrm{ng} / \mathrm{ml})$. El nivel medio de PSA después de un seguimiento de 22 meses fue de $0,15 \mathrm{ng} / \mathrm{ml}(0-$ $12,11 \mathrm{ng} / \mathrm{ml}$ ). Un $87 \%$ de los pacientes mantuvieron unos valores de PSA constantes, inferiores a $1 \mathrm{ng} / \mathrm{ml}$. El 93,4\% de los pacientes tuvieron biopsias sin presencia de Cap en los controles de seguimiento. En cuanto a los efectos secundarios, se produjo una fístula recto-uretral después de un segundo tratamiento con HIFU en un paciente con antecedentes de hemicolectomía y fístulas anales recidivantes. El $12 \%$ de los pacientes fueron sometidos a resección transuretral prostática tras el HIFU por obstrucción urinaria, aunque los autores refieren ausencia completa de casos de incontinencia urinaria importante (grados 2-3). El 47.3\% de los pacientes reconocen mantenimiento de la función eréctil. Finalmente, los autores no objetivan cambios significativos pre/post-tratamiento en el International Prostate Symptom Score (IPSS) y en el Indice de Calidad de Vida.

Blana concluye que estos resultados demuestran la eficacia y baja morbilidad asociada del HIFU. Este grupo plantea las teóricas y potenciales ventajas de la técnica como tratamiento inicial en los casos de Cap no candidatos a prostatectomía radical. Nuevamente se evidencia el poder destructivo tumoral local de la técnica (> 90\% de biopsias de control negativas). Estos autores plantean una cuestión interesante en su discusión: la definición de supervivencia libre de enfermedad. Ellos aplican 2 criterios distintos. Cuando utilizan el publicado por Amling en Journal of Urology en $2001^{11}$, con un punto de corte de PSA de $0,4 \mathrm{ng} / \mathrm{ml}$, obtienen una SLE de $71,5 \%$ a 35 meses. Sin embargo, cuando el punto de corte aplicado es el utilizado por Critz en 2002 $(0,2 \mathrm{ng} / \mathrm{ml}$, la SLE en el mismo período cae a 54\%.

¿Cuál es el mejor criterio de seguimiento en ausencia de clínica y/o biopsia positiva?. La definición de recidiva bioquímica de la Asociación Americana de Radiología y Oncología (ASTRO) (3 incrementos consecutivos de PSA tras el tratamiento radical), publicado en $1997^{12}$, ha sido puesto en duda por Horwitz ${ }^{13}$ quien ha planteado que sobrestima la SLE comparativamente a otros 
criterios. Como hemos referido anteriormente, son múltiples los puntos de corte de PSA publicados en las distintas series de la literatura en los últimos años para explicar el concepto de SLE. Esta es una de las cuestiones actualmente más controvertidas en el seguimiento del Cap tras tratamientos radicales. De los pacientes con recidiva bioquímica tras cirugía radical ${ }^{10}$, sólo el 4\% murieron de forma directamente relacionada con el tumor. Cuando el tratamiento aplicado había sido la radioterapia ${ }^{10}$, el $10 \%$ de muertes en los pacientes con progresión bioquímica fueron atribuibles directamente a la neoplasia. El valor clínico de la progresión bioquímica es ciertamente confuso, aunque sí constituye un punto final (end-point) adecuado para los ensayos clínicos. La historia natural de la progresión bioquímica puede ser realmente larga. El PSA nadir puede ser un factor pronóstico importante para la evolución del paciente, determinado especialmente a los 3 meses del procedimiento; sin embargo ningún valor constituye un criterio absoluto para justificar el inicio de un tratamiento adyuvante. La realización de biopsias de control es completamente necesaria para verificar la eficacia local del tratamiento, e indicar si es necesario llevar a cabo nuevas sesiones de $\mathrm{HIFU}^{14}$.

Es difícil interpretar el valor real de las terapias emergentes, especialmente aquéllas que cuentan con seguimientos limitados en el tiempo. Este es el caso del ultrasonido de alta intensidad. Sí parece indudable la capacidad de destrucción tumoral local. Chaussy y Thüroff presentaron recientemente dos trabajos que documentan este aspecto. Se trata de revisiones de series propias de casos, aunque con unos criterios de inclusión estrictos, un seguimiento de más de 5 años y un número considerable de pacientes incluidos (más de 500). En el primero de estos artículos ${ }^{15}$ se evalúa la eficacia histopatológica de la técnica. Ésta puede resultar en un 90\% de porcentaje de biopsias negativas a 5 años en pacientes con enfermedad de bajo y mediano riesgo. En relación al estadio tumoral, unos porcentajes actuariales a 5 años de biopsias negativas de 78\% y 60\% en la enfermedad localmente avanzada y diseminada subraya la potencia coagulativa local de la técnica. Esta importante capacidad destructiva tumoral parece depender según estos mismos autores en un segundo trabajo del esquema terapéutico y de la dosis administrada de ultrasonido ${ }^{16}$. Existe un efecto tisular directamente relacionado con la dosis en la coagulación transrectal de la próstata. Cuando la estrategia inicial intenta tratar toda la glándula (RTU prostática+HIFU completo) o tras dos sesiones consecutivas, se obtienen mejores resultados. El porcentaje de retratamientos, la estabilidad del PSA y el PSA nadir dependen directamente del esquema inicial de tratamiento (Tabla 3).

El segundo grupo de trabajos que hemos identificado se refieren a la indicación del ultrasonido de alta intensidad como terapia de rescate tras radioterapia radical (RR). Esta técnica $(R R)$ puede resultar en curación de los pacientes afectados por Cap localizado en, aproximadamente, un tercio de los $\operatorname{casos}^{17}$. El 39\% de los pacientes tratados con RR presentarían recidiva bioquímica tras una media de 2,9 años desde el tratamiento y el 23\% podrían fallecer unos 5 años tras la progresión del PSA. La estimación del porcentaje de muerte cáncer-específica 5 años tras la progresión de PSA ha sido calculada en 24\%, 40\% y $59 \%$ en casos con una puntuación de Gleason respectivamente, de $\leq 6,3+4$ o $4+3$ u 8-10 respectivamente $^{18}$. El planteamiento terapéutico en estos pacientes es bastante dispar. La prostatectomía radical de rescate puede conseguir porcentajes de SLE a 10 años aceptables $(43 \%)^{19}$. Sin embargo, la aparición de complicaciones secundarias es alta, con una incidencia de incontinencia urinaria de más de 40\%. Otras técnicas utilizables serían la braquiterapia, con SLE de $34 \%$ a 5 años aunque con menos efectos secundarios que la cirugía ${ }^{20}$. La crioterapia ofrece resultados controvertidos, que dependerían mucho del autor evaluado ${ }^{21}$. No existe evidencia científica de calidad significativa sobre la mejor terapia de rescate en la recidiva local post-RR. Nuevamente la literatura adolece de ensayos clínicos randomizados, series amplias que comparen técnicas con seguimientos largos.

En cuanto a la utilización del HIFU como terapia de rescate en recidivas locales post-RR hemos seleccionado un trabajo de Gelet publicado recientemente en Urology ${ }^{9}$. Se trata de una serie

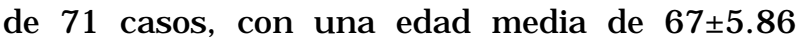


Tabla 3

Porcentaje de biopsias negativas-PSA nadir medio-estabilidad de PSA-velocidad media de PSA y porcentaje de retratamiento tras terapia con Ultrasonido de Alta Intensidad en distintos esquemas terapéuticos en cáncer prostático

\begin{tabular}{|c|c|c|c|c|c|c|c|c|c|c|c|c|}
\hline \multirow[t]{2}{*}{$N=430$} & \multicolumn{2}{|c|}{$\begin{array}{l}\text { HIFU } \\
\text { Mono }\end{array}$} & \multicolumn{2}{|c|}{$\begin{array}{c}\text { TURP+ } \\
\text { HIFU }\end{array}$} & \multicolumn{2}{|c|}{ partial } & \multicolumn{2}{|c|}{ complete } & \multicolumn{2}{|c|}{$\begin{array}{l}\text { nerve- } \\
\text { sparing }\end{array}$} & \multicolumn{2}{|c|}{$\begin{array}{l}\text { no nerve- } \\
\text { sparing }\end{array}$} \\
\hline & A & B & A & $\mathrm{B}$ & A & B & A & B & A & B & A & B \\
\hline $\begin{array}{l}\text { negative } \\
\text { biopsy } \\
\text { rate }(\%)\end{array}$ & 78.1 & 86.8 & 84.1 & 93.8 & 79.5 & 88.4 & 83.1 & 91.2 & 66.7 & 92.1 & 81.9 & 88.6 \\
\hline $\begin{array}{l}\text { median } \\
\text { nadir PSA } \\
(\mathrm{ng} / \mathrm{ml})\end{array}$ & 0.2 & 0.0 & 0.0 & 0.0 & 0.2 & 0.1 & 0.0 & 0.0 & 1.2 & 0.0 & 0.0 & 0.0 \\
\hline $\begin{array}{l}\text { PSA } \\
\text { stability } \\
\text { rate (\%) }\end{array}$ & 78.1 & 81.4 & 82.9 & 82.4 & 78.5 & 81.6 & 82.8 & 81.8 & 75.0 & 83.3 & 80.0 & 81.6 \\
\hline $\begin{array}{l}\text { median } \\
\text { PSA } \\
\text { velocity } \\
\text { (ng/ml/y) }\end{array}$ & 0.66 & 0.27 & 0.15 & 0.03 & 0.62 & 0.29 & 0.15 & 0.00 & 0.91 & 0.34 & 0.35 & 0.12 \\
\hline $\begin{array}{l}\text { HIFU } \\
\text { retreatment } \\
\text { rate }(\%)\end{array}$ & 40.1 & & 7.7 & & 32.5 & & 9,4 & & $32-3$ & & 21.8 & \\
\hline
\end{tabular}

(HIFU mono: monoterapia HIFU, TURP+HIFU: RTU próstata + HIFU, partial: HIFU parcial, complete: HIFU completo, nerve sparing: HIFU con respecto de fascículos neurovasculares; no-nerve-sparing: HIFU sin respecto de FNV). De: Chaussy C, Thuroff S, Kiel HJ. Transrectal High-intensity focused ultrasound for prostate cancer: impact of the energy dose. European Urology Supplements February 2004; 3 (2): 213.

años. El volumen prostático medio transrectal fue de $21,4 \pm 11,1 \mathrm{~cm} 3$. La puntuación de Gleason pre-HIFU: 2-6 $(\mathrm{n}=24) / 7(\mathrm{n}=13) / 8-10$ $(n=34)$. No hubo casos con diseminación a distancia objetivada previamente al HIFU. El 66,2\% de los casos presentaron Cap moderadamente/mal diferenciados. Se administraron 85 sesiones en 71 pacientes ( 1,2 sesiones/paciente). El seguimiento medio fue de 14,8 meses (6-86). $\mathrm{El}$ valor de PSA nadir determinado a 3 meses tras el tratamiento con HIFU fue de $1,97 \mathrm{ngrs} / \mathrm{ml}$.

En cuanto a los resultados, los autores presentan aspectos muy interesantes. El 80\% de los pacientes presentaron biopsias prostáticas de control negativas. Sin embargo, 40 casos (56\%) mostraron elevación de PSA sérico. De este grupo sólo se objetivó mediante biopsia cáncer residual prostático en 14; los 26 restantes ofrecieron elevación aislada de PSA con biopsia prostática negativa. La SLE, por tanto, fue determinada a 30 meses en base a 2 criterios. En primer lugar, cuando únicamente se tenían en cuenta criterios histológicos era de 73\%. Cuando además se incluían criterios bioquímicos (progresión de PSA), ese valor se reducía al 37\%.
Las reflexiones que hacen los autores, a modo de conclusiones, plantean al ultrasonido de alta intensidad como una terapia de rescate útil en la recidiva local del Cap tras RR, con unos porcentajes altos de control local de la enfermedad. Esta técnica ofrece una morbilidad aceptable comparativamente con otras alternativas. La limitación, sin embargo, de este trabajo, al igual que en series similares tratadas con otras técnicas, es la dificultad que implican estos pacientes para conseguir diagnosticar el estadio tumoral real, así como para conocer el significado clínico real a largo plazo de la progresión bioquímica y su influencia pronóstica cuando se utiliza para determinar la SLE.

\section{CONCLUSIONES}

A la vista de lo revisado en la Literatura, y apoyados por las reflexiones del Comité Oncológico de la Asociación Francesa de Urología ${ }^{22}$ podemos plantear las siguiente conclusiones:

1. Hay pocos estudios que evalúen la eficacia y morbilidad del HIFU.

2. Los seguimientos son cortos: no es posible actualmente valorar la eficacia en términos de curación definitiva. 
3. Según el Estudio Multicéntrico Europeo ${ }^{8}$ HIFU podría ser una alternativa válida en el Cap bien y moderadamente diferenciado, con PSA $<15$ ngrs $/ \mathrm{ml}$ y esperanza de vida de 10-15 años.

4. En Cap con riesgo bajo/moderado, HIFU puede conseguir resultados similares a otras terapias. En tumores de alto riesgo, NO PUEDE CONSIDERARSE COMO MONOTERAPIA (sí en tratamientos combinados).

5. Como terapia de rescate, HIFU ofrece resultados prometedores. Sus ventajas: baja morbilidad, posibilidad de retratamiento, radioterapia administrable tras fracaso

La evidencia presentada para el Ultrasonido de Alta Intensidad en la Literatura actualmente es aún de baja calidad. ¿Qué se necesita para conseguir una evidencia futura de calidad alta?:

1. Estudios randomizados y controlados con seguimiento suficiente para medir beneficios en términos de supervivencia global, $\mathrm{Q}$ o L (balance efectos adversos / beneficios). Comparaciones con terapias estándar

2. Identificación de factores pronósticos

3. Estudios de calidad de vida ( $\mathrm{C}$ o L) que evalúen pacientes sanos frente a pacientes en tratamiento

4. Conseguir diseñar estudios en los que se relacionen objetivos y supervivencia

5. Adopción de definiciones estándar para los eventos adversos, así como para los criterios de curación/fracaso terapéutico, SLE.

\section{REFERENCIAS}

1. Blana A, Walter B, Rogenhofer S, Wieland WF. High-intensity focused ultrasound for the treatment of localized prostate cancer: 5-year experience. Urology 2004;63:297-300.

2. Hummel S, Paisley S, Morgan A, Curie E, Brewer N. Clinical and cost-effectiveness of new and emerging technologies for early localised prostate cancer: a systematic review. Health Technology Assessment 2003;7(33).

3. Herranz Amo F, Arias Funez F, Arrizabalaga Moreno A, Calahorra Fernández FJ, Carballido Rodríguez J, Diz Rodríguez $\mathrm{R}$, et al. El cáncer de próstata en la Comunidad de Madrid en el año 2000. I-Incidencia. Actas Urologicas Españolas 2003;27(5):323-334.

4. Huber P, Debus J, Jenne J. Therapeutic ultrasound in tumor therapy: principles, applications and new developments. Radiologe 1996;36:64-71,

5. Chapelon JY, Margonari J, Vernier F. In-vivo effects of highintensity ultrasound on prostatic adenocarcinoma Dunning R3327. Cancer Res 1992;52:6353-6357.

6. Chaussy CG, Thüroff S. High-Intensity focused ultrasound in localized prostate cancer. J Endourol 2000;14:293-299.

7. Critz FA. A standard definition of disease freedom is needed for prostate cancer: undetectable prostate specific antigen com- pared with the American Society of Therapeutic Radiology and Oncology consensus definition. J Urol 2002;167:1310-1313.

8. Thüroff S, Chaussy C, Vallancien G, Wieland W, Kil HJ, Le Duc A, Desgrandchamps F, De la Rossette J, Gelet A. HighIntensity Focused Ultrasound and Localized Prostate Cancer: Efficacy Results from the European Multicentric Study. Journal of Endouology 2003;17(8):673-677.

9. Gelet A, Chapelon JY, Poisonier L, Bouvier R, Rouvière O, Curiel L, Janier M, Vallancien G. Local recurrence of prostate cancer after external beam radiotherapy: early experience of salvage therapy using high-intensity focused ultrasonography. Urology 2004;63:625-629.

10. Moul JW. Variables in predicting survival based on treating "PSA-only" relapse. Urologic Oncology 2003;21:292-304.

11. Amling CL, Bergstrahl EJ, Blute ML. Defining prostate specific antigen pogresion after radical prostatectomy: what is the most appropiate cut point?. J Urol 2001;165:1146-1151.

12. Consensus Statement: guidelines for PSA following radiation therapy. American Society for Therapeutic Radiology and Oncology Consensus Panel. Int $\mathrm{J}$ Radiat Oncol Biol Phys 1997;37:1035.

13. Horwitz EM, Uzzo RG, Hanlon AL. Modifying the American Society fo therapeutic radiology and oncology definition of biochemical failure to minimize the influence of backdating in patients with prostate cancer treated with 3-dimensional conformal radiation therapy alone. J Urol 2003;169:2153-2159.

14. Gelet A, Chapelon JY, Bouvier R, Rouviere O, Poissonier L, Pasticier G, Dubernard JM. Transrectal focused ultrasound and localized prostate cancer: may the nadir PSA predict the treatment success?. European Urology Supplements 2004; 3(2):213.

15. Thüroff S, Kiel HJ, Chausy C. Local histopathological efficacy of HIFU. European Urology Supplements 2004;3(2):213.

16. Chaussy C, Thüroff S, Kiel HJ. Transrectal High Intensity Focused Ultrasound for Prostate Cancer: impact of the energy dose. European Urology Supplements 2004;3(2):213.

17. Stephenson RA. Population-based prostate cancer trends in the PSA-era: data from the surveillance, epidemiology and end results program. Mongr Urol 1998;19:1-19.

18. D'Amico AV, Cote K, Loffredo M. Pretreatment predictors of time to cancer specific death after prostate specific antigen failure. J Urol 2003;169:1320-1324.

19. Amling CL, Lerner SE, Martin SK. Desoxyribonucleic acid ploidy and serum prostate speciic antigen predict outcome following salvage prostatctomy for radiation refractory prostat cancer. J Urol 1999;161:857-862.

20. Grado GL, Collins JM, Kriegshauser JS. Salvage brachitherapy for localized prostate cancer after radiotherapy failure. Urology 1999;53:2-10.

21. Pisters LL, von Eschenbach AC, Scott SM. The efficacy and complications of salvage criotherapy of the prostate. J Urol 1997; 157:921-925.

22. Rebillard X, Davin JL, Soulie M, Comité de Cancerologie de 1'Associacion Francaise d'Urologie. Progr Urol 2003;13(6): 1428-1456.

Dr. E. Lledó García

Servicio de Urología

Hospital General Universitario Gregorio Marañón

C/ Doctor Esquerdo, 46

28007 Madrid

(Trabajo recibido el 6 septiembre de 2004) 